\title{
Effect of MWCNT Filler on Properties and Flux of Chitosan/ PEG based Nanocomposites Membranes
}

\author{
Fitri Khoerunnisa ${ }^{1 *}$, Hendrawan ${ }^{1}$, Dwi Rizki Primastari ${ }^{1}$, and Riska Agiawati ${ }^{1}$ \\ ${ }^{1}$ Department of Chemistry, Indonesia University of Education, Setiabudi 229 Bandung, Indonesia 40154
}

\begin{abstract}
Biopolymer are expected to be environmentally compatible and to have great potential application as membranes material. The chitosan-poly (ethylene glycol)/PEG based composite membranes was successfully synthesized via inversed phase method. The effect of multiwalled carbon nanotubes (MWCNT) as nanofiller on properties and performances of composite membranes were intensively evaluated. The membrane was prepared by mixing of chitosan and PEG solutions at the same composition ratio while MWCNT amount in the mixture was varied. The synthesized membrane was characterized by means of FTIR spectroscopy, scanning electron microscopy (SEM), contact angle, and tensile strength measurement. The performance of composite membrane on filtration was evaluated in term of flux (permeability) and rejection (rejection) tests. The results showed that the optimum volume ratio of composite membrane solution was found at 30:10:7.5 for chitosan/ PEG/ MWCNT, respectively, as indicated by the largest flux. Insertion of MWCNT nanofiller notably enhanced hydrophilicity, porosity, and mechanical properties of composites membranes that are confirmed by contact angle, SEM images and elongation forces value, respectively. The MWCNT nanofiller remarkably increased both of flux and rejection of composite membranes up to $60 \mathrm{Lm}^{2} \mathrm{~h}^{-1}$ and $96 \%$, respectively. The remarkable enhancement of composite membrane performance is attributed to the effective interaction of MWCNT with polymeric matrix.
\end{abstract}

\section{Introduction}

The availability of clean water has become a global problem because of the continuously increasing costs of energy and increasing scarcity of water resources [1]. To address the undeniable need of pure water, various water treatment technologies have been proposed and applied at experimental and industrial levels [2]. Filtration techniques by membranes have been deemed more cost and energy effective than other separation methods $[1,3]$. The ideal membrane system should possess an excellent stability under a wide range of processing conditions, high selectivity and produce a high mass flux with minimal driving force [4-6]. To achieve this goal, both the composition and structure of the membrane are crucial. Different kinds of membranes from polymers are such potential materials and membrane technology is one such technology at the first line of decontamination and purification techniques [7-10]. During the last few years, continues development of membrane materials for water purification from bio based and renewable polymers, like chitosan have attracted great interests [11] since it is abundantly available as natural sources [12], low cost, compatibility, and has limited impact to the environment during processes and degradation [13].

\footnotetext{
Corresponding author: fitri@upi.edu
}

Application of biopolymer chitosan based membrane has the shortage on water purification due to its low mechanical properties and porosity. Many efforts have been reported on modification of membrane performance. Copolymerization of chitosan with other polymers i.e. poly (ethylene glycol)/ PEG, were frequently reported [14]. On the other hand, use of nanotechnology for membrane fabrication is a new promising method to improve membrane performance through incorporation of nanomaterials [15]. The addition of nanoparticles can alter the membrane morphology, improve permeability and reduce fouling properties of membranes, due to their high specific surface area, chemical stability and feasible functionalization [16,17]. Nanocomposite membranes can be improved by assembling nanoparticles into membranes or blending them with organic or inorganic additives [18]. Some membranes were fabricated using silica, graphite, metal oxide nanoparticle and carbon nanotubes to increase membrane permeability, and fouling resistance [19-21].

Multiwalled carbon nanotubes (MWCNTs), on the other hand, have attracted significant attention as a new type of nanofillers due to their unique structures and properties [22]. The incorporation of MWCNTs into polymeric materials has been examined in many 
applications to enhance the mechanical, thermal, and chemical stabilities of polymer matrixes in sever conditions such as high temperature and strongly oxidizing environment, and also to improve the separation properties of membranes based on the synergism between the organic-inorganic components properties [23]. A theoretical study [24] predicts a rapid mass transport in MWCNTs due to smooth nanotube walls, which may be several orders of magnitude higher than in many other porous fillers, making MWCNTs an ideal candidate filler material for membranes. MWCNTincorporated membranes have been reported to have extraordinarily high transport rate to liquid water $[24,25]$.

In this study, chitosan/PEG/MWCNT based composite membrane was synthesized by phase inversion method. The effect of MWCNT's concentration in the casting solution during preparation process on the properties and performance (flux) of membrane were intensively studied.

\section{Experimental method}

\subsection{Materials}

All chemicals used in the experiments were of reagent grade and all organic solvents were HPLC grade. The MWCNTs (95\% of purity) with 20 to $50 \mathrm{~nm}$ in outer diameter were obtained from Wako Chemical, Japan. The impurity of MWCNT was removed through acid treatment. Chitosan (molecular weights of 300.000; deacetylation degree of $85 \%$ ) and PEG/ poly ethylene glycol (mol. wt. of 6000), acetic acid, and sodium hydroxide were purchased from Merck.

\subsection{Preparation of chitosan/PEG/MWCNT membrane}

Chitosan/PEG/MWCNT filtration membrane was prepared via phase inversion method. The compositions of MWCNT was varied are shown in Table 1. Chitosan (3wt\%) and PEG $(2 w t \%)$ solutions were prepared in acetic acid $(0.1 \mathrm{M})$. MWCNT dispersion $(1 w t \%)$ was prepared in graphite oxide (GO) colloid. The optimum composition of chitosan/ PEG was followed the preceeding result [14].

Table 1. Cast solutions composition

\begin{tabular}{cccc}
\hline $\begin{array}{c}\text { Sample } \\
\text { description }\end{array}$ & $\begin{array}{c}\text { Chitosan } \\
(\mathrm{mL})\end{array}$ & $\begin{array}{c}\text { PEG } \\
(\mathrm{mL})\end{array}$ & $\begin{array}{c}\text { MWCNT } \\
(\mathrm{mL})\end{array}$ \\
\hline M0 & 30 & 10 & 0 \\
M1 & 30 & 10 & 2.5 \\
M2 & 30 & 10 & 5.0 \\
M3 & 30 & 10 & 7.5 \\
M4 & 30 & 10 & 10 \\
\hline
\end{tabular}

MWCNT dispersion, chitosan and PEG solutions were homogeneously stirred for $30 \mathrm{~min}$ under heating at $50^{\circ} \mathrm{C}$. Hereafter, the mixtures were cooling down at room temperature $\left(25^{\circ} \mathrm{C}\right)$ for $10 \mathrm{~min}$. The mixtures were further casted on PTFE template and dried at room temperature. The dried membranes was removed from the template and immersed in $\mathrm{NaOH}$ solution (1 M) for 3 $\mathrm{h}$, then rinsed with aquadest until obtaining neutral $\mathrm{pH}$, and further dried at room temperature for characterization and performance tests in ultrafiltration processes.

\subsection{Membrane characterization}

The FTIR spectra of chitosan/PEG/MWCNT nanocomposite membrane with different MWCNT contents were determined between 4000 and $400 \mathrm{~cm}^{-1}$ using Shimadzu FTIR spectrometer. Each spectrum was collected by cumulating 40 scans at a resolution of 4 wavenumbers.

A Bruker X-ray diffractometer was used to study the solid-state morphology of the chitosan/ PEG/ MWCNT nanocomposite membranes. The XRD patterns were recorded at a diffraction angle $(2 \theta)$ of from 5 to 60 to examine the crystal structure.

A scanning electron microscope (SEM-JEOL) directly provides the visual information of the cross-sectional morphology of the membrane.

Membrane hydrophobicity was quantified by measuring the contact angle that was formed between the membrane surface and water. Contact angles were measured using the sessile drop method by using a laboratory-constructed camera apparatus (JVC-TK 1270). One $\mathrm{mL}$ water droplets were placed at different positions of the membrane surface for replicates. The average value of at least five measurements was reported.

The overall porosity of all the membranes was determined using a gravimetric method defined in Eq. (1) [26]

$\varepsilon=\left(\frac{W_{w e t}-W_{d r y}}{A \times l \times \rho_{w}}\right)$

Here $W_{\text {wet }}$ is the weight of the membrane immersed in pure water for 3 days, $W_{d r y}$ is the weight of the membrane dried in a vacuum oven at temperature $80^{\circ} \mathrm{C}$ for $12 \mathrm{~h}, \mathrm{~A}$ is the membrane effective area $\left(\mathrm{m}^{2}\right), l$ is the membrane thickness $(m)$ and $\rho_{w}$ is the water density $\left(0.998 \mathrm{~g} / \mathrm{cm}^{3}\right)$.

\subsection{Performance test of membrane}

The flux (permeability) and selectivity (rejection) tests were conducted to obtain the information of membrane capability in separation and purification processes with dead-end filtration method. In particular, the flux of membranes was measured by insertion of water into filtration cell (diameter of filtration cell was $4.5 \mathrm{~cm}$, with an effective area of $15.90 \mathrm{~cm}^{2}$ ) with a stable stirring speed of $200 \mathrm{rpm}$. Prior to filtration experiment, each membrane was compacted at $0.20 \mathrm{MPa}$ for $30 \mathrm{~min}$ to reach a stable flux, then the pressure was adjusted to $0.10 \mathrm{Mpa}$. The flux measurement was conducted for 75 
min. The pure water flux $J\left(\mathrm{Lm}^{-2} \mathrm{~h}^{-1}\right)$ was measured under steady state condition through measuring the permeate water volume and calculated as the following equation (2) [27]

$$
J=\frac{V}{A \times \Delta t}
$$

where $V(\mathrm{~L})$ is the volume of permeated water, $A\left(\mathrm{~m}^{2}\right)$ is the efficient membrane area and $\Delta t(\mathrm{~h})$ is the permeation time. The flux of feed solution was recorded as $J\left(\mathrm{Lm}^{-2}\right.$ $\mathrm{h}^{-1}$ ) determined by the water quantity penetrating the membranes.

Meanwhile, the membranes rejection efficiency (selectivity) was measured using the same filtration cell. The stirred filtration cell was refilled rapidly with model foulant feed solution (dyes solution of $300 \mathrm{ppm}$ ) and the pressure of $0.1 \mathrm{MPa}$ was applied during measurement the feed and the permeate concentrations was measured via UV spectrophotometer (UV-Vis, Shimadzu) at 644.5 $\mathrm{nm}$ and calculated according to equation (3) [28]

$$
\% R=\left(1-\frac{C_{p}}{C_{f}}\right) \times 100 \%
$$

where $C_{p}$ and $C_{f}$ are the concentration of foulant in permeate and feed solutions, respectively.

\section{Results and Discussion}

\subsection{Optimum composition of MWCNT filler}

The optimum composition of MWCNT filler in membrane matrix was determined from permeability/flux of membrane. Figure 1 shows the flux of nanocomposite membranes at difference MWCNT composition.

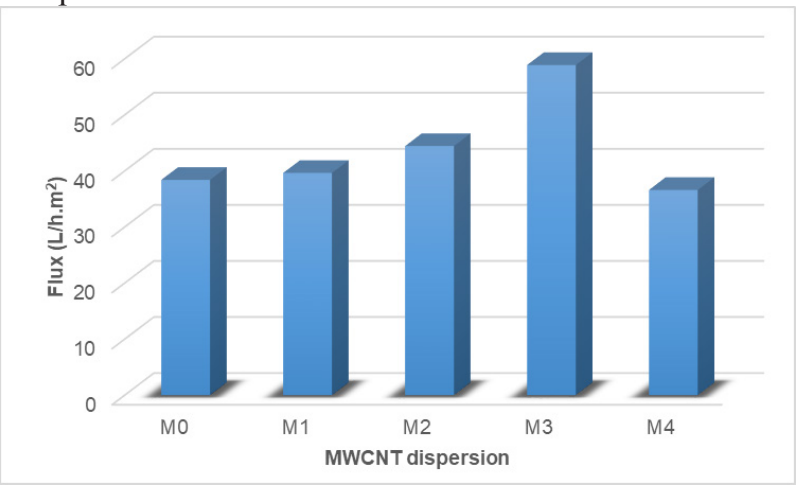

Fig 1. Flux of nanocomposites membrane at different MWCNT composition

The addition of MWCNT proportionally increases permeability of membrane composites where the optimum composition of chitosan/PEG/MWCNT based nanocomposites membrane is achieved at M3 (flux of 59 L/ h. $\mathrm{m}^{2}$ ). This may caused by several factors i.e. (1) presence of hydrophilic groups $(-\mathrm{OH}$ and $-\mathrm{COOH})$ that enable facilitate the interaction between filler and functional moeities of polymer matrix, (2) enhancement of hydrophilicity of membrane surface thus reduces fouling and consequently increase flux membrane [29], (3) MWCNT effectively inserted into the pore of membrane matrix and created matrix network (nanochannel) through pore connection for water transport [30], and (4) specific interaction of MWCNT with polymer functional groups due to charges modification [31].

However, the addition of MWCNT beyond the optimum composition leads to the reduction in membrane permeability due to aggregation of MWCNT bundles, thus blocking of membrane pore [32].

\subsection{Morphological characterization}

\subsubsection{Fourier-transform infrared spectroscopy (FTIR)}

Fourier-transform infrared spectroscopy (FTIR) was used to identify the interaction of functional groups of membrane precursors. Figure 2 demonstrates the FTIR spectra of membranes at optimum composition.

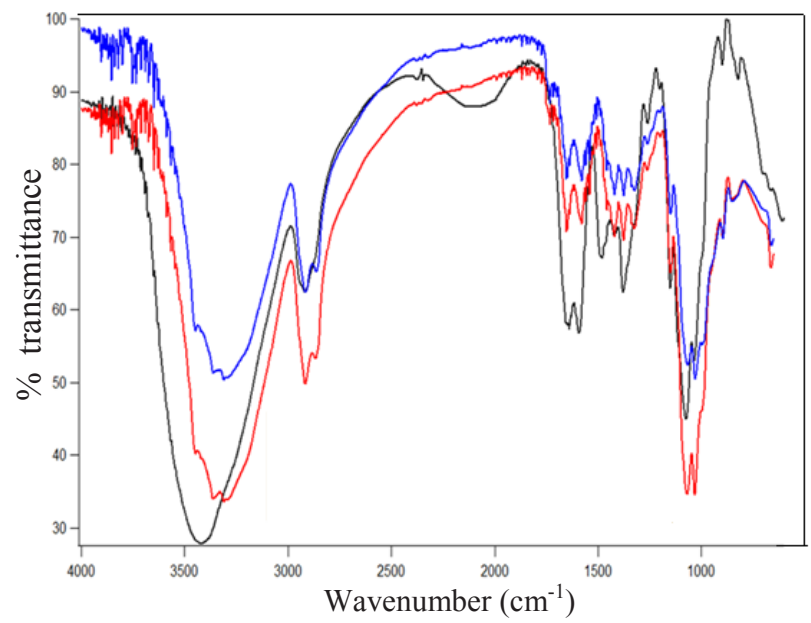

Fig 2. FTIR spectra of chitosan (black), chitosan-PEG (red), and chitosan/PEG/MWCNT nanocomposites membrane at optimum composition (blue).

The FTIR spectra of chitosan/PEG membrane and Chitosan/PEG/MWCNT membrane (Figure 2) shows absorption bands at 3446; 3365; $3299 \mathrm{~cm}^{-1}$ that sequencely indicate stretching vibration of hydoxyl O-H, stretching vibration of amine $\mathrm{N}-\mathrm{H}$, and $\mathrm{N}-\mathrm{H} / \mathrm{O}-\mathrm{H}$ polimerization vibration (Coates, 2000). Absorption bands at 2918 dan $2872 \mathrm{~cm}^{-1}$, respectively, show asymmetric stretching vibration of methylene $\left(-\mathrm{CH}_{2}\right)$ and symmetric stretching vibration of methyl $\left(-\mathrm{CH}_{3}\right)$, these finding was also supported by the presence of absorption bands at $1045-1018 \mathrm{~cm}^{-1}$ which indicates a contribution of PEG crystal's C-O bending vibration (Wang et al., 2007). Meanwhile, chitosan presence is shown by an abrorption band around $1640-1500 \mathrm{~cm}^{-1}$ that refer to amide II (N-H) vibration. There are a little band shifts from wavenumber of 3292 to $3304 \mathrm{~cm}^{-1}$ that might be caused by an increase in number of intermolecular hydrogen bonds, as an interaction between $(\mathrm{O}-\mathrm{H} / \mathrm{N}-\mathrm{H})$ of chitosan and functional groups in MWCNT. The 
decrease in absorption band intensity may be caused by the decrease in energy absorbed by functional groups as a consequence of MWCNT interaction with Chitosan/PEG copolymeric matrix.

\subsubsection{X-Ray Diffraction(XRD)}

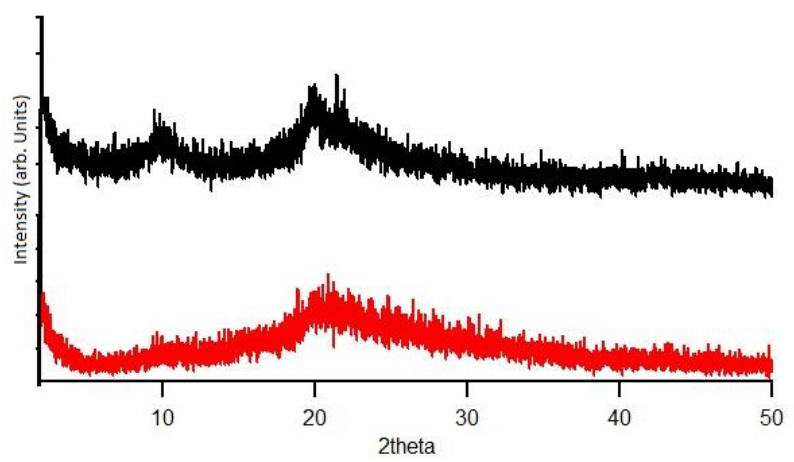

Fig 3. X-ray diffraction patterns of Chitosan/PEG membrane (red) and Chitosan/PEG/MWCNT nanocomposites membrane (black)

The X-ray diffraction pattern of Chitosan/PEG membrane demonstrates the broad peak at $2 \theta=19.9^{\circ}$ $21.8^{\circ}$, indicating the predominant amorf structure of that copolymer. The addition of MWCNT into chitosan/PEG membrane notably increases the peak intensity owing to insertion of MWCNT filler into polymeric matrix. Additional, the new peak at small angle scattering $(2 \theta=$ $10^{\circ}$ ) originated from intertube interaction of MWCNT (interlayer distance) bundles (Figure 3).

\subsubsection{Tensile Strength}

MWCNT addition significantly increased Chitosan/PEG membrane mechanical strength (Rahimipour et al., 2012) as indicated by the increasing \% elongation at break and maximum force $(\mathrm{g})$ of the membrane after MWCNT addition (Table 3). The increase in mechanical strength of copolymer through addition of MWCNT is due to formation of hydrogen bonds between Chitosan $\left(-\mathrm{NH}_{2}\right)$ and MWCNT functional groups (-COOH and $-\mathrm{OH})$.

\begin{tabular}{|c|c|c|}
\hline Sample & $\begin{array}{c}\text { Elongation } \\
\text { at Break } \\
\mathbf{( \% )}\end{array}$ & $\begin{array}{c}\text { Maximum } \\
\text { force (g) }\end{array}$ \\
\hline Chitosan/PEG & 52.07 & 1060,95 \\
\hline $\begin{array}{c}\text { Chitosan/PEG/M } \\
\text { WCNT }\end{array}$ & 53.40 & 1103,74 \\
\hline
\end{tabular}

Table 3. Tensile strength of Chitosan/PEG and Chitosan/PEG/MWCNT membranes.

\subsection{Surface morphology of membrane nanocomposites}

\subsubsection{Cross-sectional SEM images and porosity}

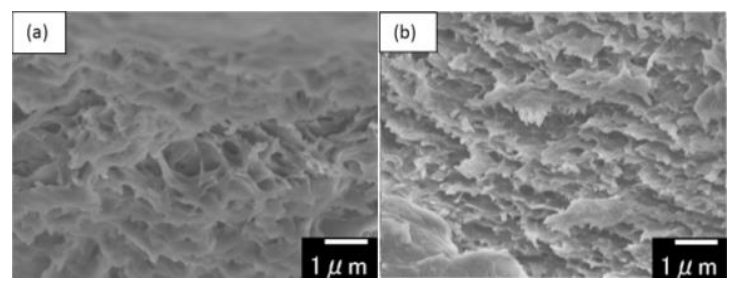

Fig 4. SEM cross-sectional images of (a) Chitosan/PEG and (b) Chitosan/PEG/MWCNT nanocomposite membranes.

Figure 4 shows the cross-sectional images of the membranes with and without the addition of MWCNT. In general, both of membranes have heterogeneous and asymmetrical porous stucture pores. Addition of MWCNT nanofiller induces the expansion of pore size and interlayer distance of copolymeric matrix, due to interconnection of polymer with MWCNT (Tang et al., 2009). The modification of pore size of Chitosan/PEG membrane by MWCNT insertion was verified by measurement of average pore radius (Figure 5). and membrane porosity (Figure 6). The improvement of pore size of copolymer membrane is owing to MWCNT addition into the membrane matrix.

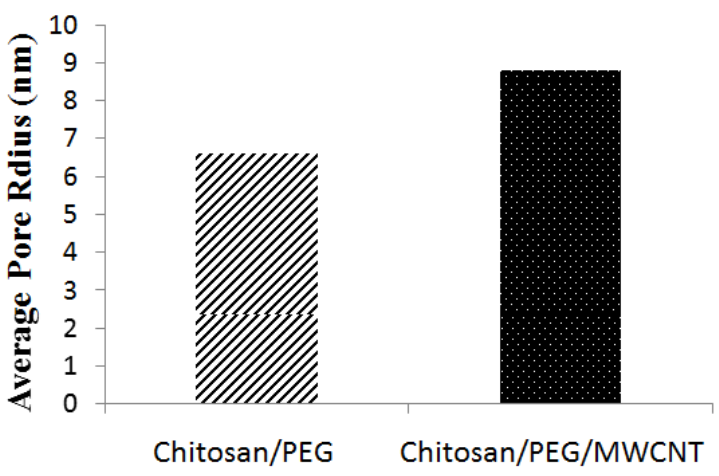

Fig 5. Average pore radius of the membranes (left) Chitosan/PEG and (right) Chitosan/PEG/MWCNT

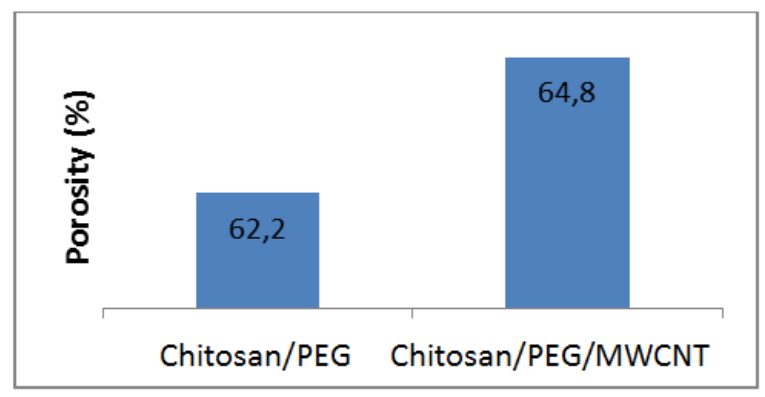

Fig 6. Porosity of Chitosan/PEG (left) and Chitosan/PEG/MWCNT (right) membranes 


\subsubsection{Contact Angle}
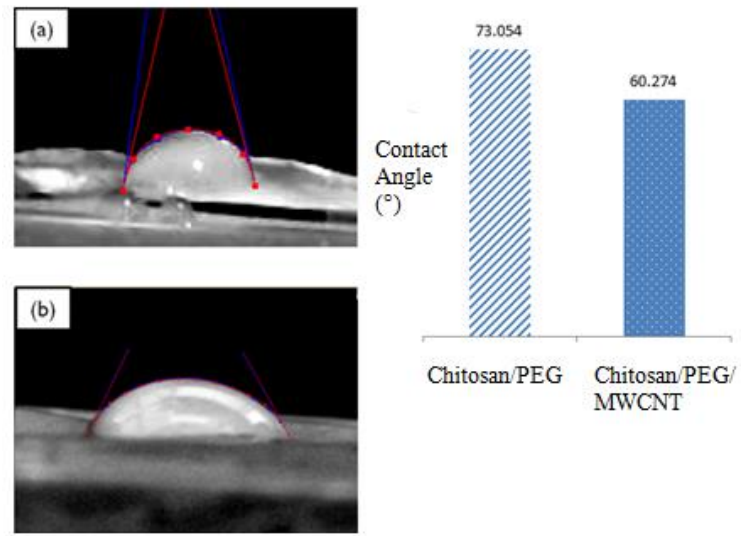

Fig 7. Contact angle of Chitosan/PEG (a) and (b) Chitosan/PEG/MWCNT membranes

Membrane hydrophilicity was tested using sessile drop test to measure the membrane surface contact angle. As shown inFfigure 7 MWCNT addition decreases the membrane contact angle, in other word increases the membrane hydrophilicity. The membrane hydrophilicity was increased as an effect of higher porosity due to MWCNT addition (Figure 6). The functional groups that present in MWCNT (-COOH, -OH) also contribute to increase the membrane hydrophilicity (Vatanpour et al., 2012).

\subsection{Permeability and Perselectivity}

\subsubsection{Pure Water Flux}
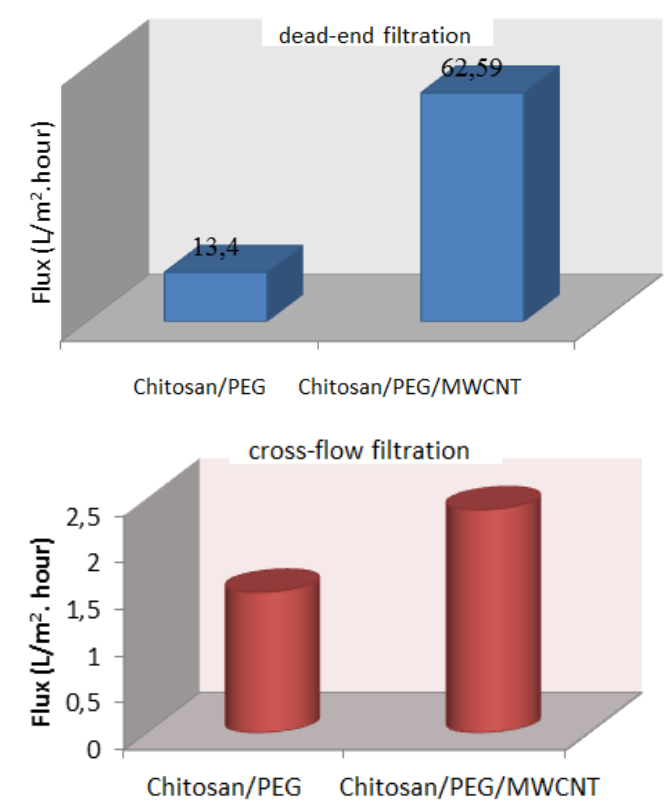

Fig 8. Pure water flux of Chitosan/PEG membrane (left) and Chitosan/PEG/MWCNT nanocomposite membrane (right) at 2 atm and membrane thickness of $0,08 \mathrm{~mm}$, using dead-end (top) and cross-flow (bottom) filtation system.
Permeability of the membranes is measured as flux value ( $\mathrm{L} / \mathrm{m}^{2}$ hour). The permeability of Chitosan/PEG/ MWCNT membrane was increased sharply as shown in figure 8. The membrane flux increased because of membrane pore expansion and increase in hydrophilicity due to the addition of MWCNT. Membrane permeability heavily depend on membrane thickness and pressure applied. Figure 9 shows flux value at varied pressure and membrane thickness. Increasing pressure lead to an increase in flux value, while increasing membrane thickness lead to a decrease in flux value (Rahimipour et al., 2012; Zhao et al., 2013; Daraei et al., 2012). The highest flux was obtained using the thinnest membrane $(0,07 \mathrm{~mm})$, and at highest pressure (3,6 atm).

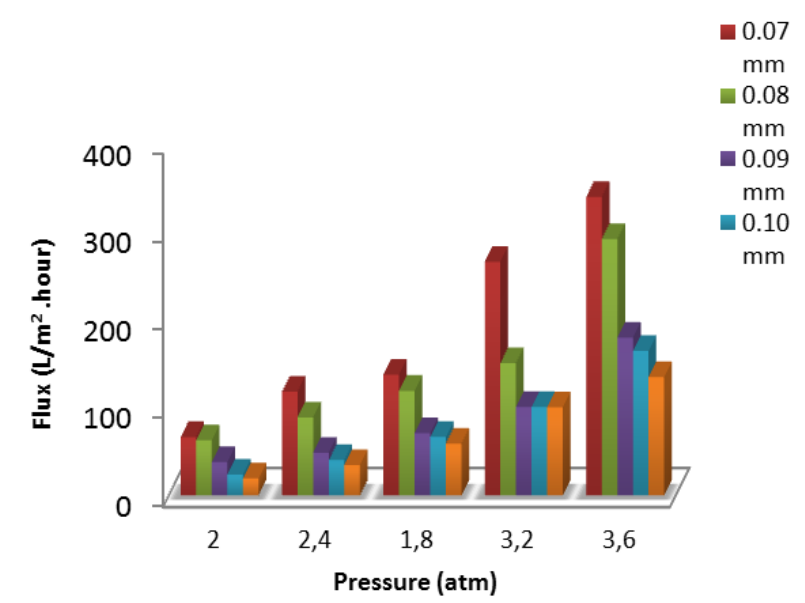

Fig 9. Pure water flux of Chitosan/PEG/MWCNT nanocomposites membrane at varied pressure and membrane thickness using dead-end filtation system.

\subsubsection{Membrane selectivity}

Selectivity is measured to understand the membrane's ability to reject species of certain size and is written as a $\%$ rejection $(\mathrm{R})$.

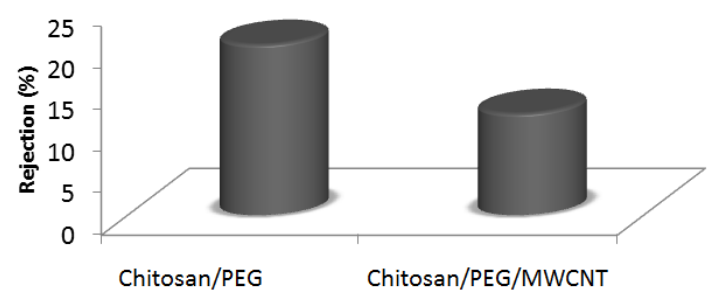

Fig 10. Protein rejection of the Chitosan/PEG membrane and Chitosan/PEG/MWCNT nanocomposites membrane.

Figure 10 shows protein rejection of the membranes. It can be seen that Chitosan/ PEG/ MWCNT nanocomposites membrane. did not reject protein as much as Chitosan/PEG membrane. This slight decrease in protein selectivity is due to the increase in average pore radius in Chitosan/PEG/MWCNT nanocomposites membrane. 


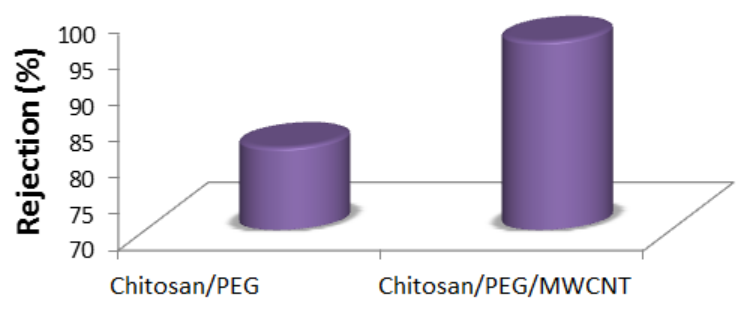

Fig 11. Wantex pigment rejection of the Chitosan/PEG membrane and Chitosan/PEG/MWCNT nanocomposites membrane.

Meanwhile Chitosan/PEG/MWCNT nanocomposites membrane yield higher wantex pigment rejection (Figure 11). This can be explained by the ability of MWCNTs to absorb pigments such as wantex pigment, due to its high surface area and poroeus structure Gupta et al. (2013).

\section{Conclusion}

Based on the results, it can be concluded that Chitosan/PEG/MWCNT nanocomposite membrane had successfully synthesized. The MWCNT successfully incorporated into the membrane matrix shown by FTIR, XRD, and SEM characterization. MWCNT addition to Chitosan/PEG membrane sharply increases membrane premeability and selectivity against pigments as well as increasing its mechanical properties. The nanocomposites membrane can be applied at high pressure to be classified as an ultrafiltration membrane.

\section{References}

1. M.A. Shannon, P.W. Bohn, M. Elimelech, J.G. Georgiadis, B. J. Marĩnas and A. M. Mayes, Nature, 452, 301 (2008)

2. V.K. Gupta, I. Ali, T.A. Saleh, A. Nayak, S. Agarwal, RSC Advances 2 (16), 6380-6388 (2012).

3. M. Elimelech and W. A. Phillip, Science, 333, 712 (2011).

4. D. Li and H. Wang, J. Mater. Chem., 20, 4551 (2010).

5. M.M. Pendergast, E.M. Hoek, Energy Environ. Sci. 4 (6), 1946-1971, (2011).

6. R. Khalil, E. Sheha, T. Hanafy, \& O., Al-Hartomy, Materials Express, 4(6), 483-490 (2014).

7. S. He, Y. Lin, Z. Wei, L. Zhang, J. Lin, \& S. Nazarenko, Polymers for Advanced Technologies, 26(4), 300-307(2015).

8. C.S. Ferreira, S.G. Caridade, J.F., Mano, \& N.M. Alves, Polymers for Advanced Technologies, 25(12), 1492-1500 (2014).

9. N. Akther, A. Sodiq, S. Giwa, H. Daer, A. Arafat, \& S.W. Hasan, Chemical Engineering Journal, 281, 502-522 (2015).

10. J. Li, J., Gao, G. Sui, L. Jia, C. Zuo, \& Q. Deng, Materials Express, 4(6), 491-498 (2014).
11. V.K. Thakur, A.S. Singha, \& B.N. Misra, Journal of Applied Polymer Science, 122(1), 532-544. (2011).

12. S.I., Voicu, A.I.V. MuhuletAntoniac, \& M.S. Corobea. Key Engineering Materials, 638, 27-30. (2015).

13. F. Khoerunnisa, Hendrawan, Y. Sonjaya, A. Putri, Synthesis and Characterization of composites filtration membranes based chitosan-poly(ethylene glycol), AIP conference proceedings 1699, 040009 (2015)

14. Z. Fan, Z. Wang, M. Duan, J. Wang, S.Wang, J. Membrane Sci. 310 (1-2), 402-408 (2008).

15. T. Wu et al,. RSC Adv., 5(11), pp.7880-7889. (2014).

16. E. Celik, L. Liu \& H. Choi, Water Research, 45(16), 5287-5294. (2011).

17. X. Qu, P.J.J. Alvarez, Q. Li, Water Res. 47, 39313946, (2013).

18. Liang S. et al., J. Membr. Sci. 394, pp. 184-192. (2012).

19. A.H. Cui et al. J. Membr. Sci. 360, pp. 259-264. (2010)

20. A .Bottino G.A. Capannelli Desalination 146, pp. 35-40. (2002).

21. S. Kar, R.C. Bindal, P.K. Tewari, Nano Today 7, 385-389 (2012)

22. D.Q. Vu, W.J. Koros, J. Membrane Science 211, 311-334 (2003).

23. A.I. Skoulidas, D.M. Ackerman, J.K. Johnson, D.S. Sholl, Rapid transport of gases in carbon nanotubes, Phys. Rev. Lett. 89, 18590 (2002)

24. J.K. Holt, H.G. Park, Y. Wang, M. Stadermann, A.B. Artyukhin, C.P. Grigoropoulos, A. Noy, O. Bakajin, Science 312, 1034-1037 (2006)

25. M. Majumder, N. Chopra, R. Andrews, B.J. Hinds, Nature 438, 44 (2005). 